\title{
Observation of Various and Spontaneous Magnetic Skyrmionic Bubbles at Room Temperature in a Frustrated Kagome Magnet with Uniaxial Magnetic Anisotropy
}

\author{
Zhipeng Hou, Weijun Ren, * Bei Ding, Guizhou Xu, Yue Wang, Bing Yang, Qiang Zhang, \\ Ying Zhang, Enke Liu, Feng Xu, Wenhong Wang,* Guangheng Wu, Xixiang Zhang, \\ Baogen Shen, and Zhidong Zhang
}

The quest for materials hosting topologically protected skyrmionic spin textures continues to be fueled by the promise of novel devices. Although many materials have demonstrated the existence of such spin textures, major challenges remain to be addressed before devices based on magnetic skyrmions can be realized. For example, being able to create and manipulate skyrmionic spin textures at room temperature is of great importance for further technological applications because they can adapt to various external stimuli acting as information carriers in spintronic devices. Here, the first observation of skyrmionic magnetic bubbles with variable topological spin textures formed at room temperature in a frustrated kagome $\mathrm{Fe}_{3} \mathrm{Sn}_{2}$ magnet with uniaxial magnetic anisotropy is reported. The magnetization dynamics are investigated using in situ Lorentz transmission electron microscopy, revealing that the transformation between different magnetic bubbles and domains is via the motion of Bloch lines driven by an applied external magnetic field. These results demonstrate that $\mathrm{Fe}_{3} \mathrm{Sn}_{2}$ facilitates a unique magnetic control of topological spin textures at room temperature, making it a promising candidate for further skyrmion-based spintronic devices.
Dzyaloshinskii-Moriya interaction (DMI) is active. A number of intriguing electromagnetic phenomena, including the topological Hall effect, ${ }^{[7]}$ skyrmion magnetic resonance, ${ }^{[17]}$ thermally induced ratchet motion, ${ }^{[18]}$ and effective magnetic monopoles, ${ }^{[19]}$ have been demonstrated to be closely related to the topologically nontrivial spin texture of skyrmions. These novel topological properties, together with nanoscale dimensions, a stable particlelike feature, and an ultralow threshold for current-driven motion, make magnetic skyrmions fundamentally promising for applications in next-generation high-density and low-dissipation memory devices. ${ }^{[1-5]}$ However, although a very recent publication by Tokunaga et al. ${ }^{[20]}$ reported the observation of skyrmion lattices above room temperature (RT) in $\beta$-type $\mathrm{Cu}-\mathrm{Zn}$ Mn alloys, skyrmions in the bulk chiral magnets have been mostly observed below $\mathrm{RT}^{[6-16]}$ Moreover, the spin texture of skyr-

The quest for materials hosting topologically protected nanometric spin textures, so-called magnetic skyrmions or magnetic skyrmionic bubbles, continues to be fueled by the promise of novel devices. ${ }^{[1-5]}$ The skyrmionic spin textures have been mostly observed in noncentrosymmetric crystals, such as the cubic chiral magnets $\mathrm{MnSi},{ }^{[6-9]} \quad(\mathrm{Mn}, \mathrm{Fe}) \mathrm{Ge}^{[10,11]} \mathrm{FeCoSi}{ }^{[12]}$ $\mathrm{Cu}_{2} \mathrm{OSeO}_{3},{ }^{[13-15]}$ and also the polar magnet $\mathrm{GaV}_{4} \mathrm{~S}_{8},{ }^{[16]}$ where mions that were stabilized by DMI in chiral magnets is quite limited. The skyrmions with variable spin textures may be more attractive for further technological applications because they can adapt to various external stimuli acting as information carriers in spintronic devices. Therefore, one particularly important current research direction aims at the discovery of new materials that host skyrmions with variable spin textures at room temperature.
Dr. Z. P. Hou, Dr. B. Ding, Dr. Y. Wang, Dr. Y. Zhang, Dr. E. K. Liu, Prof. W. H. Wang, Prof. G. H. Wu, Prof. B. G. Shen

Beijing National Laboratory for Condensed Matter Physics Institute of Physics

Chinese Academy of Sciences

Beijing 100190, China

E-mail: wenhong.wang@iphy.ac.cn

Dr. W. J. Ren, Dr. B. Yang, Prof. Z. D. Zhang

Shenyang Materials Science National Laboratory

Institute of Metal Research

Chinese Academy of Sciences

72 Wenhua Road, Shenyang 110016, China

E-mail:wjren@imr.ac.cn

\author{
Dr. G. Z. Xu, Prof. F. Xu \\ School of Materials Science and Engineering \\ Nanjing University of Science and Technology \\ Nanjing 210094, China \\ Dr. Q. Zhang, Prof. X. X. Zhang \\ King Abdullah University of Science and Technology (KAUST) \\ Physical Science and Engineering (PSE) \\ Thuwal 23955-6900, Saudi Arabia
}

DOI: 10.1002/adma.201701144 
In addition to the noncentrosymmetric chiral magnets in which the magnetic skyrmions are stabled by DMI, the centrosymmetric materials with uniaxial magnetic anisotropy (UMA) are another family of materials that can host skyrmions. ${ }^{[21-26]}$ In these materials, the competition between the magnetic dipole interaction and uniaxial easy axis anisotropy is the key force in the formation of skyrmions. ${ }^{[21-26]}$ The skyrmions in centrosymmetric materials are topologically equivalent to those in the chiral magnets ${ }^{[21-26]}$ but possess two degrees of freedom, i.e., helicity and vorticity. ${ }^{[5]}$ Since their internal degree of freedom is similar to that in the topologically trivial magnetic bubbles, they are also called skyrmionic bubbles. ${ }^{[24]}$ The most interesting physics in skyrmionic bubbles is that the vorticity of their spin textures varies with the internal structure of the Bloch lines (BLs), resulting in a variety of spin textures. ${ }^{[22-26]}$ For instance, a new type of spin texture formed by two skyrmions with opposite spin vorticity (the topological number equals 2), called a bi-skyrmion, has been experimentally discovered in the centrosymmetric tetragonal magnetite $\mathrm{La}_{1-x} \mathrm{Sr}_{x} \mathrm{MnO}_{3}(x=0.315)^{[23]}$ and hexagonal $\left(\mathrm{Mn}_{1-x} \mathrm{Ni}_{x}\right)_{65} \mathrm{Ga}_{35}(x=0.5)^{[25]}$ at temperatures around 60 and $300 \mathrm{~K}$, respectively. More recently, Yu et al. ${ }^{[24]}$ have found a variety of spin textures in magnetic skyrmionic bubbles in orthorhombic magnetite $\mathrm{La}_{1-x} \mathrm{Sr}_{x} \mathrm{MnO}_{3}(x=0.175)$ at $100 \mathrm{~K}$. Thus, the multifarious topological nature of skyrmionic bubbles offers us an opportunity to manipulate their topological spin textures through external stimuli.

Recently, the domain structures in the frustrated magnet with tunable UMA were studied numerically by Leonov et al.[27] They showed that the UMA strongly affects spin ordering. They also predicted that different spin structures, including isolated magnetic skyrmions, may coexist in a frustrated magnet, and that the isolated magnetic skyrmions also possess additional degrees of freedom (spin vorticity and helicity), similar to that in the magnetic skyrmionic bubbles. Their predictions are not only extremely interesting, but also point to further investigation of the variable topological spin textures in the frustrated magnets. Furthermore, Pereiro et al. ${ }^{[28]}$ have theoretically shown that Heisenberg and DMI interactions in kagome magnets can overcome the thermal fluctuation and stabilize the skyrmions at relatively high temperatures, even at room temperature. Based on the theoretical investigations above, we revisit the frustrated magnets with kagome lattice to search for the possible skyrmionic bubbles that are able to stabilize at room temperature.

One of the promising materials is $\mathrm{Fe}_{3} \mathrm{Sn}_{2}$, suggested by Pereiro et al., ${ }^{[28]}$ which has a layered rhombohedral structure with alternate stacking of the Sn layer and the Fe-Sn bilayers along the $c$-axis, as shown in Figure 1a. The Fe atoms form bilayers of offset kagome networks, with $\mathrm{Sn}$ atoms throughout the kagome layers as well as between the kagome bilayers. Very importantly, this material is a noncollinear frustrated ferromagnet with a high Curie temperature $T_{\mathrm{c}}$ of $640 \mathrm{~K}$, and shows a spin reorientation that the easy axis rotates gradually from the $c$ axis to the $a b$-plane as the temperature decreases. ${ }^{[29-32]}$ Recently, a large anomalous Hall effect was observed in this material, which is strongly related to the frustrated kagome bilayer of Fe atoms. ${ }^{[33,34]}$ In this communication, we report that magnetic skyrmionic bubbles with various spin textures can indeed be realized in the single crystals of $\mathrm{Fe}_{3} \mathrm{Sn}_{2}$ at room temperature.
The emergence of skyrmionic bubbles and the magnetization dynamics associated with the transition of different bubbles via the field-driven motion of the Bloch lines are revealed by in situ Lorentz transmission electron microscopy (LTEM), and further supported by the micromagnetic simulations and magnetic transport measurements. These results demonstrate that $\mathrm{Fe}_{3} \mathrm{Sn}_{2}$ facilitates a unique magnetic control of topological spin textures at room temperature, making it a promising candidate for further skyrmion-based spintronic devices.

High-quality single crystals of $\mathrm{Fe}_{3} \mathrm{Sn}_{2}$ were synthesized by a Sn-flux method, as described in the Experimental Section. These crystals are layered and exhibit mirror-like hexagonal faces with a small thickness (see Figure S1 in the Supporting Information). By using single-crystal X-ray diffraction, the crystal parameters were identified as $a=b=5.3074 \AA$ and $c=19.7011 \AA$, with respect to the rhombohedral unit cell (space group $R-3 m)$, agreeing well with the previous studies. ${ }^{[32-34]}$ Having determined the unit lattice parameters and orientation matrix, we then found that the hexagonal face was normal to [001] with the (100), (010), and (110) faces around (see Figure S2 in the Supporting Information). In addition, as shown in Figure S3 in the Supporting Information, both the temperature-dependent in-plane and out-of-plane magnetization curves measured on the bulk crystal indicate that the ferromagnetic transition temperature $T_{c}$ is about $640 \mathrm{~K}$.

Figure 1b shows the temperature-dependent UMA coefficient $\left(K_{\mathrm{u}}\right)$, which was estimated by the approximation of $K_{\mathrm{u}}=H_{\mathrm{k}} M_{\mathrm{s}} / 2$, where $M_{\mathrm{s}}$ is the saturation magnetization and $H_{\mathrm{k}}$ is the anisotropy field defined as the critical field above which the difference in magnetization between the two magnetic field directions $(H / / a b$ and $H / / c)$ becomes smaller than 2\% (see Figure S4 in the Supporting Information). One can notice that the value of $\mathrm{Ku}$ increases monotonically with the decrease of temperature. Simultaneously, the Fe moments gradually rotate from the $c$-axis toward the $a b$-plane (see the inset of Figure $1 b$ ), demonstrating a gradual transformation from the magnetically easy axis (uniaxial magnetic anisotropy) into a magnetically easy plane with decreasing the temperature. The most important message conveyed to us by Figure $1 \mathrm{~b}$ is that the magnetic domain configuration in $\mathrm{Fe}_{3} \mathrm{Sn}_{2}$ may vary over a very wide temperature range of $80-423 \mathrm{~K}$, because the domain structure is strongly affected by the magnetic anisotropy. Therefore, $\mathrm{Fe}_{3} \mathrm{Sn}_{2}$ should be a good platform for us to explore the correlation between the spin texture and $\mathrm{Ku}$ in a very wide temperature range.

We then imaged the magnetic domain structures using LTEM under zero magnetic field in the temperature range of $300-130 \mathrm{~K}$, as shown in Figure 1c-f. The corresponding selected-area electron diffraction (SAED) patterns suggest that the sample is normal to the [001] direction (see the inset of Figure 1b). Nanosized stripe domains with an average periodicity of $\approx 150 \mathrm{~nm}$ were clearly observed. Notably, the value of periodicity is comparable to that in the bulk $\left(\mathrm{Mn}_{1-x} \mathrm{Ni}_{x}\right)_{65} \mathrm{Ga}_{35}(x=0.5),{ }^{[25]}$ but nearly two times larger than that in the $\mathrm{La}_{1-x} \mathrm{Sr}_{x} \mathrm{MnO}_{3}(x=0.175) .{ }^{[24]}$ The sharp contrast between the dark stripe domains and the bright walls suggests that the domains possess out-of-plane magnetizations and are separated by Bloch domain walls. With the decrease of temperature, the stripes' periodicity widened while the domain wall 
(a)

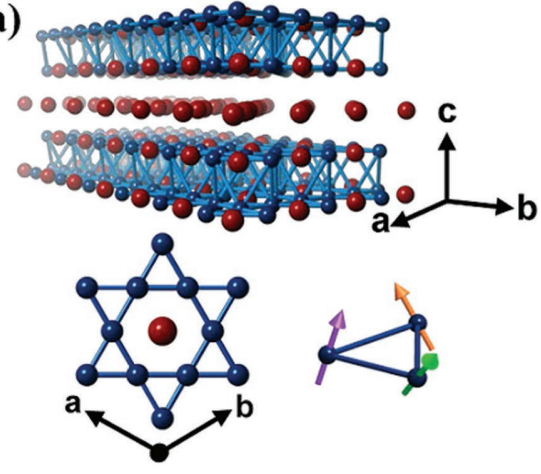

Fe• Sn॰

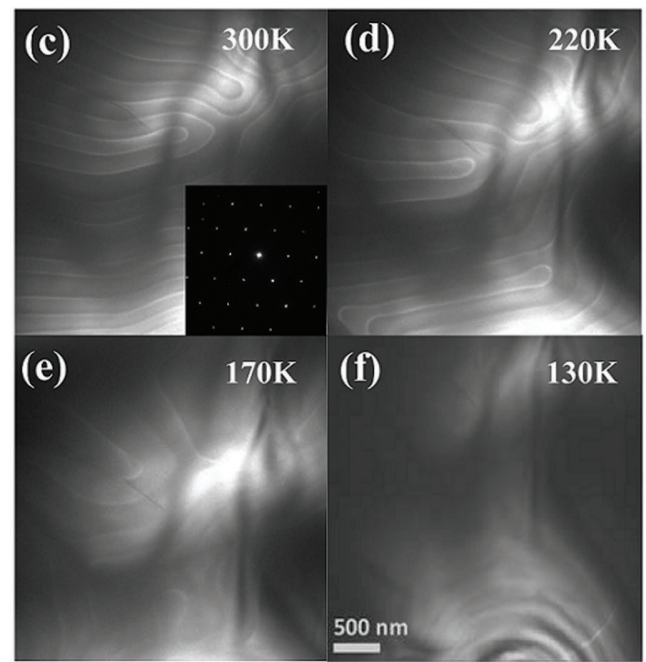

(b)
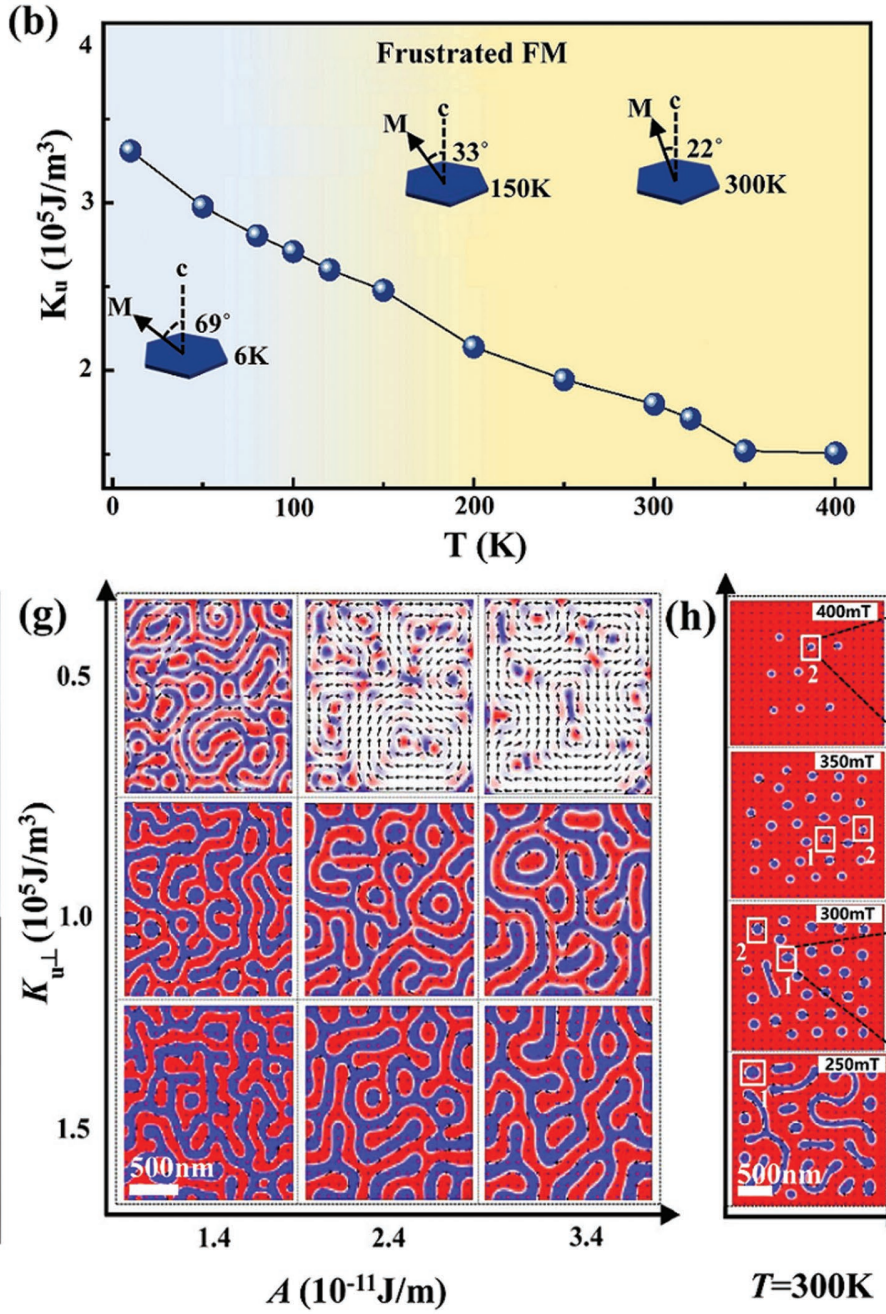

Figure 1. Structure, magnetic properties, and micromagnetic simulations of $\mathrm{Fe}_{3} \mathrm{Sn}_{2}$. a) The crystal structure of $\mathrm{Fe}_{3} \mathrm{Sn}_{2}$ (up), a top view of the kagome layer of Fe atoms (down, left) and a possible spin (arrows) configuration of the Fe atoms (down, right). b) The temperature dependence of anisotropy constant $\left(K_{u}\right)$ in the temperature range 10-400 K. The insets (from right to left) show the schematic of the angle between the magnetic easy axis and the $c$-axis at 300, 150, and $6 \mathrm{~K}$, respectively. $\mathrm{c}-\mathrm{f}$ ) The representative images of the domain structures of same area in a Fe $\mathrm{Sn}_{2}$ thin plate taken by Lorentz transmission electron microscopy (LTEM) with an electron beam perpendicular to the $a b$-plane of $\mathrm{Fe}_{3} \mathrm{Sn}_{2}$ when the sample temperature was lowered from 300 to $130 \mathrm{~K}$ in zero external magnetic field. The inset of panel (c) shows the corresponding selected-area electron diffraction (SAED) pattern. g) The plan view of equilibrium states under different ratios of exchange constant $A$ and perpendicular component for the magnetocrystalline magnitude $K_{\mathrm{u} \perp}$ for a fixed $M_{\mathrm{s}}=5.7 \times 10^{5} \mathrm{~A} \mathrm{~m}^{-1}$ and thickness $(100 \mathrm{~nm})$ in zero external magnetic field. The magnetization along the $z$-axis $\left(m_{z}\right)$ is represented by regions in red $\left(+m_{z}\right)$ and blue $\left(-m_{z}\right)$, whereas the in-plane magnetization $\left(m_{x}, m_{y}\right)$ is represented by the white regions. h) The simulated fielddependent domain morphology under several magnetic fields that capture the domain evolution from stripes to type-II bubbles and skyrmionic bubbles.

thickness $D$ remained almost a constant (see Figure S5 in the Supporting Information). Interestingly, we found that when the temperature was lower than $130 \mathrm{~K}$, the stripe domains disappeared and vortex domains formed, indicating that the spin starts to lie into the $a b$-plane below $130 \mathrm{~K}$. The critical temperature of the LTEM sample coincides with that of the bulk sample, but with a slight deviation. ${ }^{[32-34]}$ This feature suggests that the spin texture of domains in the bulk and LTEM samples show little differences. That can be attributed to the fact that the magnetic anisotropy in $\mathrm{Fe}_{3} \mathrm{Sn}_{2}$ is high enough to overcome the spin rearrangement effect resulting from the increase of demagnetizing energy in the thin LTEM sample. To understand the physics behind this domain structure transformation, we performed numerical simulations with estimated parameters of exchange constant $(A)$ and anisotropy energy $\left(K_{\mathrm{u} \perp}\right)$ associated with the perpendicular component of the anisotropy field (see the Experimental Section). As shown in Figure 1g, the stripe domain gradually transformed into a vortex domain with decreasing $K_{\mathrm{u} \perp}$, agreeing well with the LTEM images. This feature suggests that the domain morphology in $\mathrm{Fe}_{3} \mathrm{Sn}_{2}$ is mainly governed by the anisotropy perpendicular to the $a b$-plane, which is consistent with the simulated results based on a frustrated magnet. ${ }^{[27]}$ It is well known that the magnetic domain structure also depends greatly on an external magnetic field. Therefore, we have simulated the domain structure under different magnetic fields that are perpendicular to the ab-plane (as shown in Figure 1h). It is interesting to note that the stripe domains gradually transformed into bubbles with increasing 


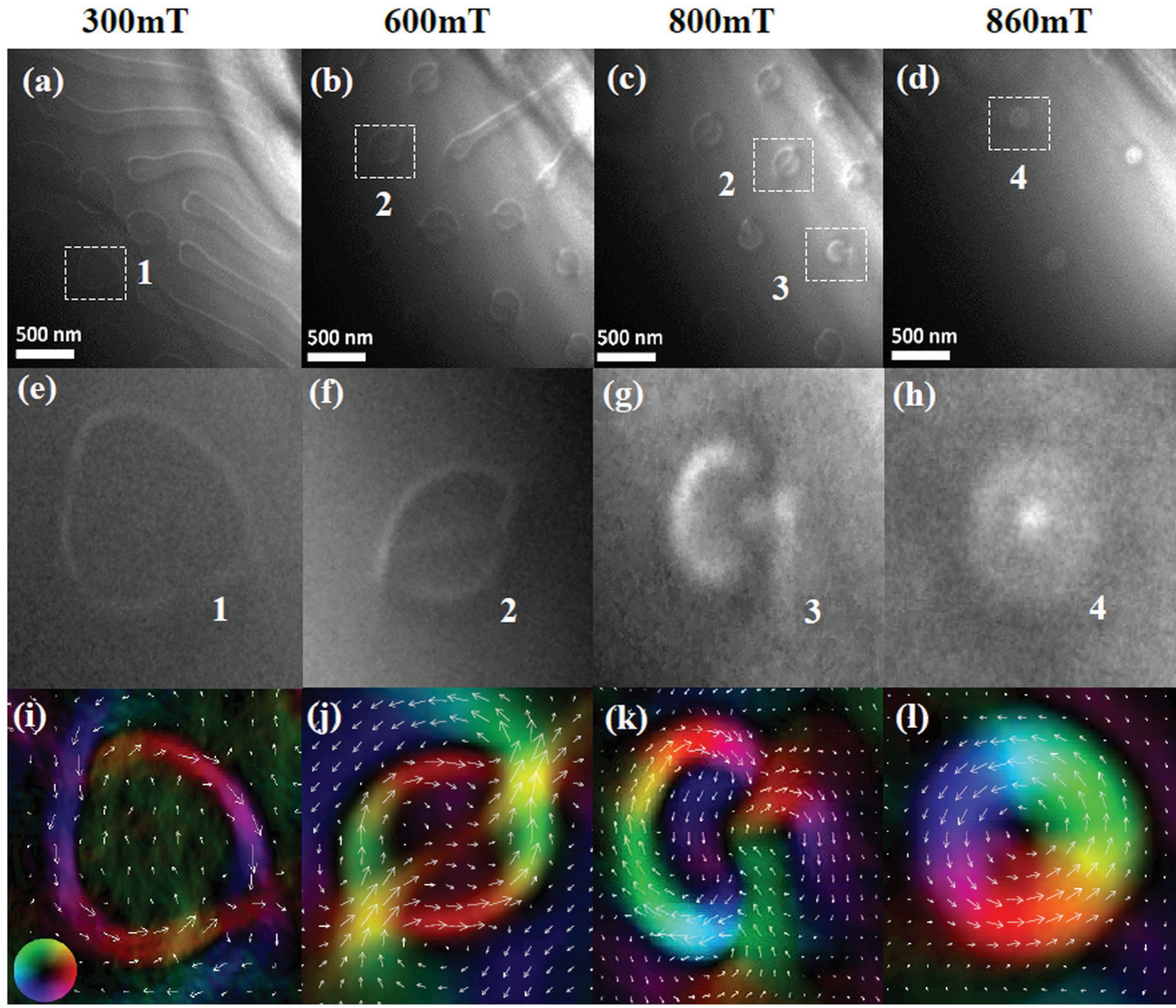

Figure 2. Magnetic field dependence of the magnetic domain morphology imaged using LTEM at $300 \mathrm{~K}$. a-d) The overfocused LTEM images under different out-of-plane magnetic fields at $300 \mathrm{~K}$. The regions in the white boxes show the different types of magnetic bubble domains. e-h) Enlarged LTEM images of the white boxes in panels (a)-(d) showing the magnetic bubble domains. i-l) Corresponding spin textures for the bubble domains shown in panels (e)-(h), extracted from the analysis using TIE. Colors (the inset of panel (i) shows the color wheel) and white arrows represent the direction of in-plane magnetic induction, respectively, whereas the dark color represents the magnetic induction along the out-of-plane direction. Panels (e) and $(\mathrm{f})$ display the type-II bubbles, and panels $(\mathrm{k})$ and $(\mathrm{l})$ show different types of skyrmionic bubbles.

the external field. More strikingly, isolated magnetic skyrmions formed when the magnetic field increased to $400 \mathrm{mT}$.

To experimentally observe the domain structure variation with an external magnetic field predicted by the simulation, we imaged the domain structure under different magnetic fields at room temperature using LTEM. Figure $2 \mathrm{a}-\mathrm{d}$ shows the overfocused LTEM images under different out-of-plane magnetic fields (the corresponding zero-field image is shown in Figure S6 in the Supporting Information). The gradual transformation from a stripe domain structure into magnetic skyrmionic bubbles is clearly observed as the magnetic field increases from 0 to $860 \mathrm{mT}$. In Figure 2a, we show a snapshot of the transformation process under a magnetic field of $300 \mathrm{mT}$. One can notice that the stripe domains, dumbbellshaped domains, and magnetic bubbles coexist in the image.
We actually observed that, during the evolution of the domain structures, the stripe domains gradually broke into the dumbbell-like domains first before evolving into the magnetic bubbles. When the magnetic field increased above $800 \mathrm{mT}$, all the stripes and dumbbell-like domains completely transformed into magnetic bubbles. One should note that we only changed the external magnetic field and kept all other conditions constant in Figure 2a-d. Therefore, the changes in domain structure can be entirely ascribed to the external field effect. A closer analysis of the entire transformation process reveals that the spin texture of the magnetic bubbles changes dramatically with increasing the magnetic field, as shown in Figure $2 \mathrm{e}-\mathrm{h}$ (the bubbles with different spin textures are notated by different numbers). The structural evolution of the magnetic bubbles with increasing external magnetic field should be closely 
related to the change of topology in the bubbles, as previously observed. ${ }^{[24,26]}$

To characterize the topological spin textures of the magnetic bubbles, a transport-of-intensity equation (TIE) was employed to analyze the over and underfocused LTEM images. Figure $2 \mathrm{i}-1$ displays the spin textures of the bubble domains shown in Figure $2 \mathrm{e}-\mathrm{h}$. The white arrows show the directions of the inplane magnetic inductions, while the black regions represent the domains with out-of-plane magnetic inductions. Bubble "1" composed of a pair of open BLs, is characteristic of the domain structure commonly observed in ferromagnetic compounds with uniaxial magnetic anisotropy. In this bubble, the topological number $N$ is determined to be 0 , because the magnetizations of the BLs are nonconvergent. Further increase of the magnetic field induced the formation of bubble " 2 " which has two arc-shaped walls with opposite helicity, separated by two BLs. Similar to that of bubble "1" the spin texture of bubble "2" is also not convergent, leading to the same topological number, $N=0$. However, when the magnetic field increased above $800 \mathrm{mT}$, the topological number of bubbles " 3 " and "4" transforms from 0 to 1 , being equal to that of skyrmions. As shown in Figure $2 \mathrm{k}$, bubble " 3 " has a pendulum structure with two BLs, in which the spin textures become convergent. Compared with the recent results obtained in $\mathrm{La}_{1-x} \mathrm{Sr}_{x} \mathrm{MnO}_{3}(x=0.175)$ by Yu et al., [24] bubble "3" can also be regarded as a specific type of rarely observed skyrmionic bubble. Bubble "4" is the most orthodox skyrmionic bubble, possessing the same domain structure as the skyrmions observed in chiral magnets. One important feature of this bubble is that the thickness of the Bloch wall is comparable to the radius of the bubble, leading to a small core region. Following previous reports, ${ }^{[24]}$ we understand that bubble " 3 " transformed into bubble "4" through the motion of BLs driven by the magnetic field. Therefore, although the magnetic textures in bubbles " 3 " and "4" are strikingly different, they are homeomorphic in topology. To understand the above transformations of spin texture in more detail, we successfully recorded the transformation process from the topologically nontrivial magnetic bubbles to the topologically protected skyrmions in a $\mathrm{Fe}_{3} \mathrm{Sn}_{2}$ (001) thin plate using in situ LTEM (see Movie in the Supporting Information). Figure 3a-f presents several snapshots of the transformation process, selected from a movie taken by LTEM at $300 \mathrm{~K}$ and under different out-of-plane magnetic fields. We observed a pair of BLs moves along the bubble wall and eventually dies out within $8.3 \mathrm{~s}$, as the field increased from 840 to $850 \mathrm{mT}$. These results demonstrate clearly that isolated magnetic skyrmions can be realized in the frustrated $\mathrm{Fe}_{3} \mathrm{Sn}_{2}$ magnet through $\mathrm{BL}$ motion by tuning the external magnetic field, even at room temperature.

In addition to the observation of various spin textures of skyrmionic bubbles during BL motion, we further found that the magnetic domain configurations after turning off the external fields depend strongly on the strength of the external fields. If the thin plate sample is first magnetized to saturation (i.e., the sample is in single-domain state), then the domain will return to the multistripe state after turning off the external magnetic field (see Figure S7 in the Supporting Information). However, if the sample is magnetized to an intermediate state (for example, at a field of $700 \mathrm{mT}$ as shown in Figure S8 in the Supporting Information), the domain structure evolves differently after turning off the external magnetic field. Figure $4 \mathrm{a}$ shows the underfocused LTEM image taken at $300 \mathrm{~K}$ after turning off the external field of $700 \mathrm{mT}$ by which the sample was magnetized to an intermediate state. The coexistence of different types of magnetic domains, e.g., magnetic bubbles, stripes,

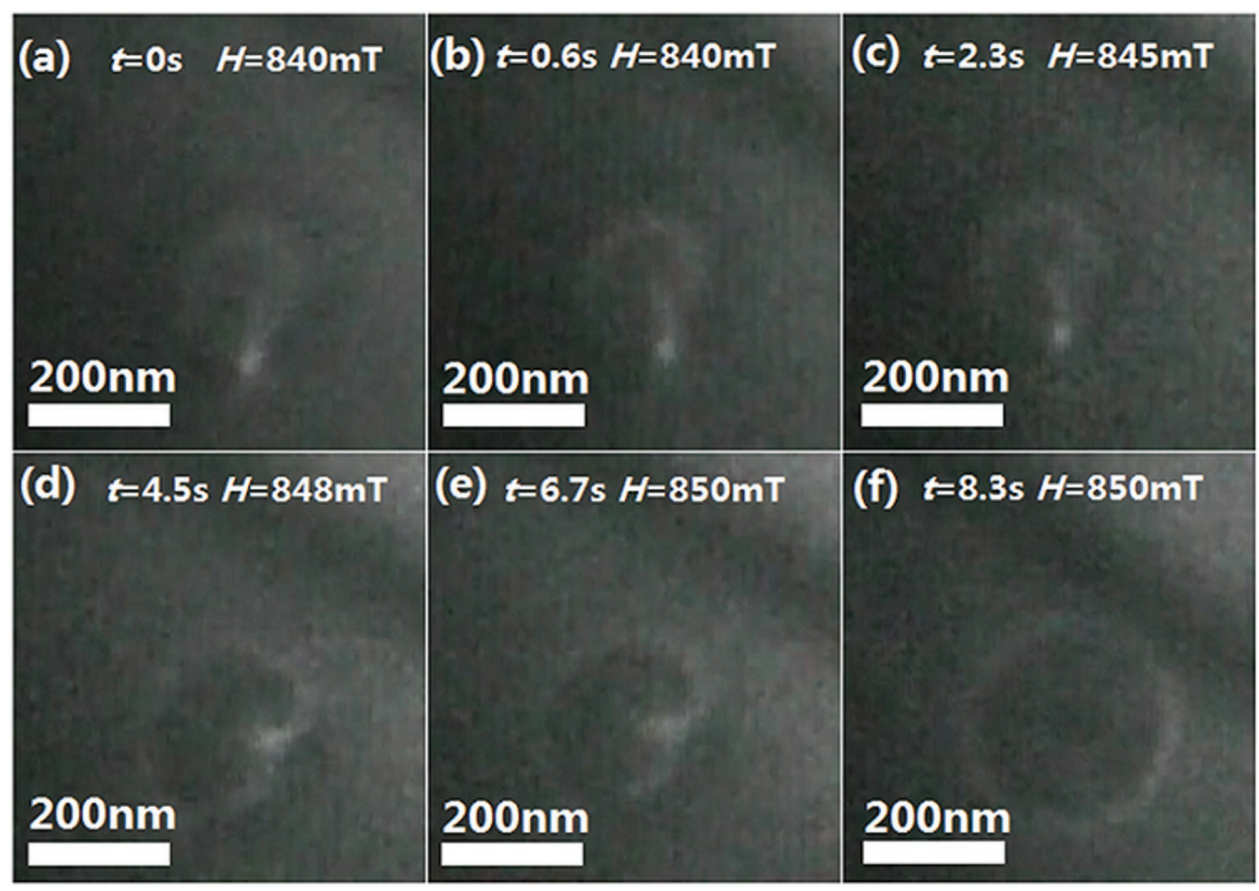

Figure 3. Evolution of the magnetic bubble through Bloch line (BL) motion, induced by the magnetic field. a- $f$ ) Series of LTEM images of bubble " 3 " observed at different times and fields applied along the $c$-axis. The field was increased slowly for $8 \mathrm{~s}$ from 840 to $850 \mathrm{mT}$. 


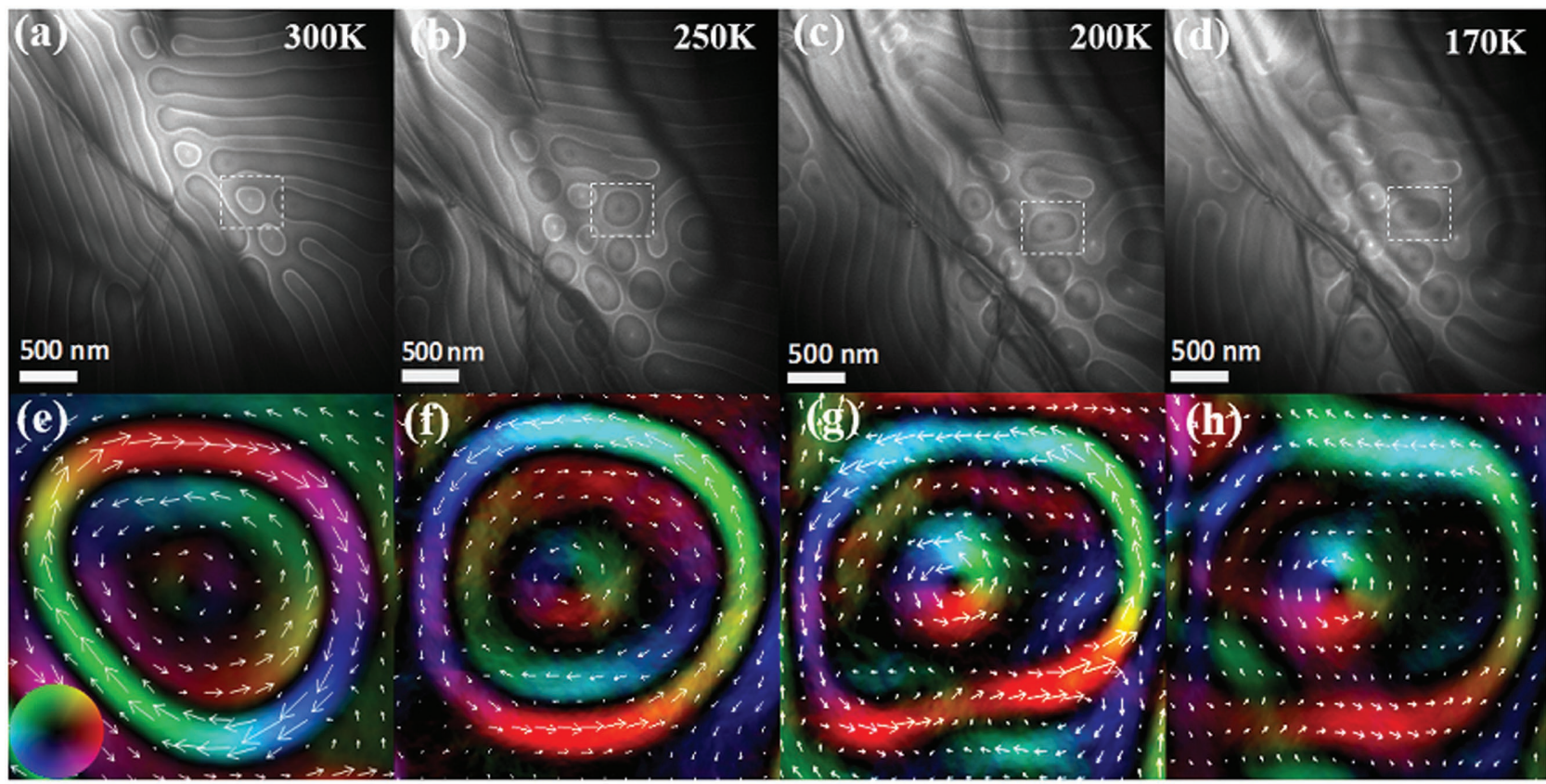

Figure 4. LTEM images of the magnetic domain structures taken at different temperatures after turning off the external magnetic field applied along $c$-axis. The field of $700 \mathrm{mT}$ is not strong enough to saturate the sample magnetically. a-d) Stripe domains and skyrmionic bubbles coexist in the sample over the temperature range of 300 to $170 \mathrm{~K}$, after turning off the magnetic field. The regions enclosed in the squares are the skyrmionic bubbles. e-h) The corresponding magnetization textures obtained from the TIE analysis for the bubble domains in the squares in panels (a)-(d). The inset of panel (e) shows the color wheel. The spontaneous skyrmionic bubbles with triple-ring structures and random helicities are observed.

and dumbbell-like domains is clearly observed. After careful analysis of the image using TIE, the detailed spin texture of the domain enclosed by the square in Figure $4 \mathrm{a}$ is shown in Figure 4e. Unexpectedly, the magnetic bubbles possess three concentric rings. It is particularly interesting that the winding directions of the inner and outer rings are opposite to that of the middle ring, indicating that the helicity reverses inside the bubbles. These spontaneous bubbles can be considered skyrmions, analogous to those observed in $\mathrm{BaFe}_{12-x-0.05} \mathrm{Sc}_{x} \mathrm{Mg}_{0.05} \mathrm{O}_{19} \cdot{ }^{\text {[22] }}$

To explore the evolution of the domain structure with temperature, the domain structures in the same region as shown in Figure 4a were also imaged at several lower temperatures without changing the external field (Figure 4b-d). These results help us to understand how the domain structure evolves under the influence of different magnetic energies, i.e., magnetic anisotropy, exchange energy, and static magnetic energy. As the temperature decreases, the size of the spontaneous bubbles changes slightly and the stripes gradually transform into skyrmions. Consequently, the number of spontaneous bubbles increases with decreasing temperature. The maximum density of the bubbles appears at $250 \mathrm{~K}$. The spontaneous bubbles vanish as the temperature further decrease below $100 \mathrm{~K}$, due to the transformation from uniaxial, out-of-plane anisotropy into in-plane anisotropy (Figure 1b). The corresponding spin textures of the skyrmions marked in Figure $4 \mathrm{~b}-\mathrm{d}$ were also extracted by using TIE analysis, as shown in Figure $4 \mathrm{f}-\mathrm{h}$. It was found that the size of the innermost ring increases with decreasing the temperature. Micromagnetic simulations are currently ongoing in order to explore the interplay among the different magnetic parameters behind these features.
The formation of magnetic skyrmionic bubbles and isolated skyrmion spin textures in the bulk $\mathrm{Fe}_{3} \mathrm{Sn}_{2}$ single crystals were further studied and confirmed by magnetic and magnetotransport measurements, similar to previous studies of other skyrmion-based materials..$^{[7,20,25,35]}$ It should be noted here that the sample for magnetic and magnetotransport measurements is from the same crystals used for LTEM observations. In Figure 5a, we show the dependence of magnetoresistance (MR) on the magnetic field $(H)$ that is applied normal to the $a b$-plane in the temperature range of 100-400 K. The inset shows the details of the MR- $H$ curve at $300 \mathrm{~K}$, in which two broad peaks are clearly seen at about 200 and $800 \mathrm{mT}$, respectively. Based on the LTEM results shown in Figure 2 and our analysis, it can be concluded that the peak at $200 \mathrm{mT}\left(H_{\mathrm{a}}\right)$ reflects the starting point of the transformation from stripes to magnetic bubbles, whereas the peak at $800 \mathrm{mT}\left(H_{\mathrm{m}}\right)$ represents the starting point of the transformation from magnetic bubbles to insulated skyrmions. When the magnetic field increases above $900 \mathrm{mT}\left(H_{\mathrm{c}}\right)$, the sample reaches the magnetically saturated state (see the $M-H$ curves in Figure S4 in the Supporting Information) in which the spins are aligned along the field direction, and consequently all the skyrmions die out. A close inspection of the MR $-H$ curves in the range of 400-100 K reveals that all three critical fields shift monotonically with temperature as indicated by the dotted lines. As shown in Figure 5b, we can also identify the three critical fields in the field-dependent AC-susceptibility, though the critical fields are slightly lower than those observed in the MR results and show slightly weaker temperature dependence. Based on the LTEM, MR, and AC-susceptibility results, we can roughly create a magnetic phase diagram as depicted in Figure 5c. Based on the phase diagram, we predict 

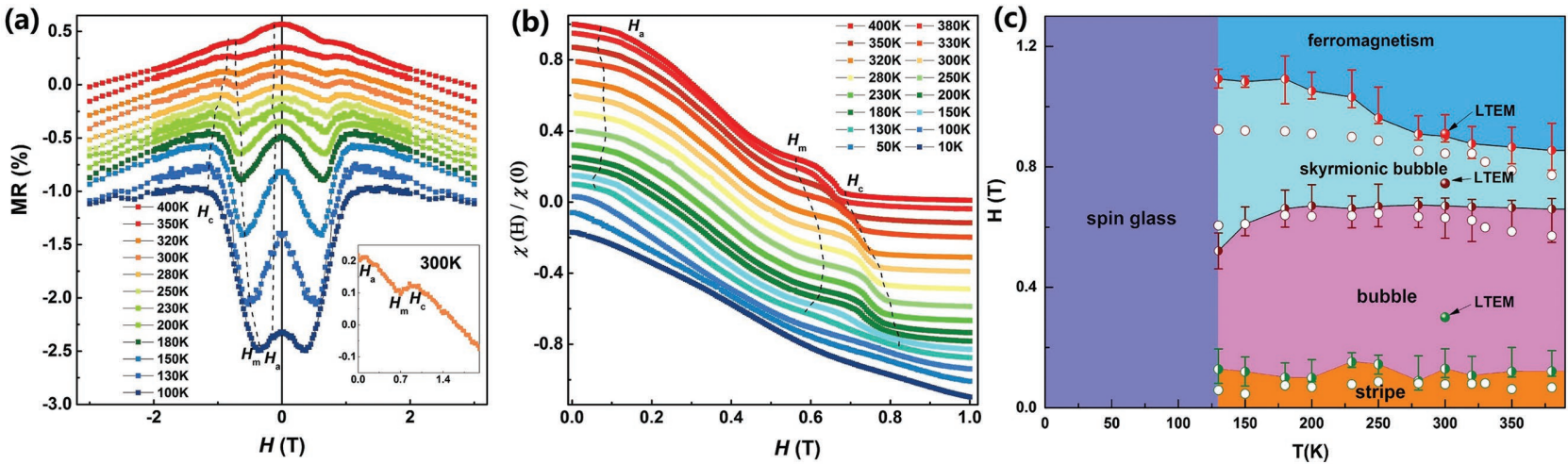

Figure 5. Field dependence of magnetoresistance (MR), AC-susceptibility, and the magnetic phase diagrams of Fe $\mathrm{Sn}_{2}$. a) The magnetic field $(H)$ dependence of MR obtained in the temperature range of 100-400 K, with magnetic fields applied normal to the $a b$-plane. The inset shows the details of the MR-H curve measured at $300 \mathrm{~K}$. b) The magnetic field dependence of the real part of AC-susceptibility $\chi$ in the temperature range of 100-400 K. c) Magnetic phase diagram of bulk sample in the magnetic field versus the temperature plane, as deduced from the temperature dependence of MR and $\chi$ curves. The three fully filled circles indicate the experimental data obtained from the in situ LTEM observations. The error bars were added based on the results measured on three different samples.

that the topological spin texture states in $\mathrm{Fe}_{3} \mathrm{Sn}_{2}$ may extend to a much higher temperature, perhaps up to Curie temperature $(\approx 640 \mathrm{~K})$. However, due to the technical limitations of our current measurements, we could not perform the experiments at a temperature higher than $400 \mathrm{~K}$. It is well known that stable skyrmionic states at high temperatures are critical for technical applications in magnetic storage and spintronics devices. Therefore, the observation of a skyrmion state in $\mathrm{Fe}_{3} \mathrm{Sn}_{2}$, not only in a very wide temperature range but also at a high temperature, strongly suggests that $\mathrm{Fe}_{3} \mathrm{Sn}_{2}$ is a very promising material for both skyrmion physics and potential technical applications of magnetic skyrmions.

The ongoing and future studies will include electrically probing various exciting phenomena in this material, such as the skyrmion Hall effect, ${ }^{[3,37]}$ and quantizing the transport of magnetic skyrmions, ${ }^{[38,39]}$ similar to the study of emergent electrodynamics of skyrmions in bulk chiral materials. ${ }^{[40]}$

\section{Experimental Section}

Sample Preparation: Single crystals of $\mathrm{Fe}_{3} \mathrm{Sn}_{2}$ were synthesized by using the $\mathrm{Sn}$-flux method with a molar ratio of $\mathrm{Fe}: \mathrm{Sn}=1: 19$. The starting materials were mixed together and placed in an aluminum crucible with higher melting temperatures at the bottom. This process was performed in a glove box filled with argon gas. To avoid the influence of volatilization of Sn at high temperatures, the whole assembly was first sealed inside a tantalum (Ta) tube under proper Ar pressure. The Ta tube was then sealed in a quartz tube filled with 2 mbar Ar pressure. The crystal growth was carried out in a furnace by heating the tube from room temperature up to $1150{ }^{\circ} \mathrm{C}$ over a period of $15 \mathrm{~h}$, holding at this temperature for $72 \mathrm{~h}$, cooling to $910^{\circ} \mathrm{C}$ over $6 \mathrm{~h}$, and subsequently cooling to $800{ }^{\circ} \mathrm{C}$ at a rate of $1.5 \mathrm{~K} \mathrm{~h}^{-1}$. The excess Sn flux was removed by spinning the tube in a centrifuge at $800{ }^{\circ} \mathrm{C}$. After the centrifugation process, most of the flux contamination was removed from the surfaces of crystals and the remaining flux was polished.

Magnetic and Transport Measurements: The magnetic moment was measured by using a Quantum Design physical properties measurement system (PPMS) between 10 and $400 \mathrm{~K}$, whereas the magnetic moment above $400 \mathrm{~K}$ was measured by using a vibrating sample magnetometer (VSM). To measure the (magneto-) transport properties, several single crystals were milled into a bar shape with a typical size of about
$0.6 \mathrm{~mm} \times 0.4 \mathrm{~mm} \times 0.05 \mathrm{~mm}$. Both longitudinal and Hall resistivity were measured using a standard four-probe method on a Quantum Design PPMS. The field dependence of the Hall resistivity was obtained after subtracting the longitudinal resistivity component.

LTEM Measurements: The thin plates for Lorentz TEM observations were cut from bulk single-crystalline samples and thinned by mechanical polishing and argon-ion milling. The magnetic domain contrast was observed by using Tecnai F20 in the Lorentz TEM mode and a JEOLdedicated Lorentz TEM, both equipped with liquid-nitrogen, lowtemperature holders $(\approx 100 \mathrm{~K})$ to study the temperature dependence of the magnetic domains. The magnetic structures were imaged directly in the electron microscope. To determine the spin helicity of the skyrmions, three sets of images with under-, over-, and just (or zero) focal lengths were recorded by a charge-coupled device (CCD) camera, and then the high-resolution in-plane magnetic induction distribution mapping was obtained by QPt software, based on the TIE. The inversion of magnetic contrast can be seen by comparing the under and overfocused images. The colors and arrows depict the magnitude and orientation of the in-plane magnetic induction. The objective lens was turned off when the sample holder was inserted, and the perpendicular magnetic field was applied to the stripe domains by increasing the objective lens gradually in very small increments. The specimens for the TEM observations were prepared by polishing, dimpling, and subsequently ion milling. The crystalline orientation of the crystals was determined by SAED.

Micromagnetic Simulations: Micromagnetic simulations were carried out with 3D object oriented micromagnetic framework (OOMMF) code, based on the LLG function. ${ }^{[4]]}$ Slab geometries of dimensions $2000 \mathrm{~nm} \times$ $2000 \mathrm{~nm} \times 100 \mathrm{~nm}$ were used, with rectangle mesh of size $10 \mathrm{~nm} \times$ $10 \mathrm{~nm} \times 10 \mathrm{~nm}$. We used a damping constant $\alpha=1$ to ensure quick relaxation to the equilibrium state. The material parameters were chosen according to the experimental values of $\mathrm{Fe}_{3} \mathrm{Sn}_{2}$, where the saturation magnetization $M_{\mathrm{s}}=5.66 \times 10^{5} \mathrm{~A} \mathrm{~m}^{-1}$ at room temperature, and the uniaxial magnetocrystalline anisotropy constant $K_{\mathrm{u}}=1.8 \times 10^{5} \mathrm{~J} \mathrm{~m}^{-3}$. As the magnetic moment aligned obliquely along the $c$-axis, we defined $K_{\mathrm{u} \perp}$ as the anisotropy energy associated with the perpendicular component of the anisotropic field, i.e., $K_{\mathrm{u} \perp}=\left(\frac{1}{2}\right) H_{\mathrm{k} \perp} M_{\mathrm{s}}$. The exchange constant $A$ was estimated to be $1.4 \times 10^{-11} \mathrm{~A} \mathrm{~m}^{-1}$ by $D=\pi \sqrt{A / K_{\mathrm{u}}}$, where $D$ is the domain wall width obtained from the LTEM results. These three parameters vary with temperature, hence we investigated the dependence of domain morphology on exchange energy $(A)$ and anisotropy energy as shown in Figure $1 \mathrm{~g}$ by fixing the $M_{\mathrm{s}}$. The equilibrium states are all obtained by fully relaxing the randomly distributed magnetization. The simulations on the field-dependent domain structures at $300 \mathrm{~K}$ (as shown in Figure 1h) were conducted 
at zero temperature (no stochastic field) but the values of parameters correspond to $300 \mathrm{~K}$, because the change tendency of magnetic domain alters little by temperature.

\section{Supporting Information}

Supporting Information is available from the Wiley Online Library or from the author.

\section{Acknowledgements}

Z.P.H., W.J.R., and B.D. contributed equally to this work. The authors thank Jie Cui and Dr. Yuan Yao for discussions and their help in LTEM experiments. This work was supported by the National Natural Science Foundation of China (Grant nos. 11474343, 11574374, 11604148, 51471183, 51590880, 51331006, and 5161192), King Abdullah University of Science and Technology (KAUST) Office of Sponsored Research (OSR) under Award No: CRF-2015-2549-CRG4, China Postdoctoral Science Foundation No. Y6BK011 M51, a project of the Chinese Academy of Sciences with Grant no. KJZD-EW-M05-3, and the Strategic Priority Research Program B of the Chinese Academy of Sciences under the Grant no. XDB07010300.

\section{Conflict of Interest}

The authors declare no conflict of interest.

\section{Keywords}

$\mathrm{Fe}_{3} \mathrm{Sn}_{2}$, kagome magnets, skyrmionic bubbles, spintronic devices, topological spin textures

Received: February 27, 2017 Revised: April 23, 2017 Published online:

[1] T. H. R. Skyrme, Nucl. Phys. 1962, 31, 556

[2] K. Yamada, S. Kasai, Y. Nakatani, K. Kobayashi, H. Kohno, A. Thiaville, T. Ono, Nat. Mater. 2007, 6, 269.

[3] R. Hertel, C. M. Schneider, Phys. Rev. Lett. 2006, 97, 177202.

[4] A. Fert, V. Cros, J. Sampaio, Nat. Nanotechnol. 2013, 8, 152.

[5] N. Nagaosa, Y. Tokura, Nat. Nanotechnol. 2013, 8, 899.

[6] S. Mühlbauer, B. Binz, F. Jonietz, C. Pfleiderer, A. Rosch, A. Neubauer, R. Georgii, P. Boni, Science 2009, 323, 915.

[7] A. Neubauer, C. Pfleiderer, B. Binz, A. Rosch, R. Ritz, P. G. Niklowitz, P. Boni, Phys. Rev. Lett. 2009, 102, 186602.

[8] C. Pappas, E. Lelivre-Berna, P. Falus, P. M. Bentley, E. Moskvin, S. Grigoriev, P. Fouquet, B. Farago, Phys. Rev. Lett. 2009, 102, 197202.

[9] A. Tonomura, X. Z. Yu, K. Yanagisawa, T. Matsuda, Y. Onose, N. Kanazawa, H. S. Park, Y. Tokura, Nano Lett. 2012, 12, 1673.

[10] X. Z. Yu, N. Kanazawa, Y. Onose, K. Kimoto, W. Z. Zhang, S. Ishiwata, Y. Matsui, Y. Tokura, Nat. Mater. 2011, 10, 106

[11] H. Wilhelm, M. Baenitz, M. Schmidt, U. K. Rößler, A. A. Leonov, A. N. Bogdanov, Phys. Rev. Lett. 2011, 107, 127203.

[12] X. Z. Yu, Y. Onose, N. Kanazawa, J. H. Park, J. H. Han, Y. Matsui, N. Nagaosa, Y. Tokura, Nature 2010, 465, 901.

[13] S. Seki, X. Z. Yu, S. Ishiwata, Y. Tokura, Science 2012, 336,198
[14] T. Adams, A. Chacon, M. Wagner, A. Bauer, G. Brandl, B. Pedersen, H. Berger, P. Lemmens, C. Pfleiderer, Phys. Rev. Lett. 2012, 108, 237204.

[15] S. Seki, S. Ishiwata, Y. Tokura, Phys. Rev. B 2012, 86, 060403.

[16] I. Kézsmárki, S. Bordács, P. Milde, E. Neuber, L. M. Eng, J. S. White, H. M. Rǿnnow, C. D. Dewhurst, M. Mochizuki, K. Yanai, H. Nakamura, D. Ehlers, V. Tsurkan, A. Loidl, Nat. Mater. 2015, 14, 1116.

[17] Y. Onose, Y. Okamura, S. Seki, S. Ishiwata, Y. Tokura, Phys. Rev. Lett. 2012, 109, 037603.

[18] M. Mochizuki, X. Z. Yu, S. Seki, N. Kanazawa, W. Koshibae, J. Zang, M. Mostovoy, Y. Tokura, N. Nagaosa, Nat. Mater. 2014, 13, 241.

[19] P. Milde, D. Köhler, J. Seidel, L. M. Eng, A. Bauer, A. Chacon, J. Kindervater, S. Mühlbauer, C. Pfleiderer, S. Buhrandt, C. Schütte, A. Rosch, Science 2013, 340, 1076.

[20] Y. Tokunaga, X. Z. Yu, J. S. White, H. M. Rǿnnow, D. Morkawa, Y. Taguchi, Y. Tokura, Nat. Commun. 2015, 6, 7638.

[21] Y. S. Lin, J. Grundy, E. A. Giess, Appl. Phys. Lett. 1973, 23, 485.

[22] X. Z. Yu, M. Mostovoy, Y. Tokunaga, W. Z. Zhang, K. Kimoto, Y. Matsui, Y. Kaneko, N. Nagaosa, Y. Tokura, Proc. Natl. Acad. Sci. USA 2012, 109, 8856.

[23] X. Z. Yu, Y. Tokunaga, Y. Kaneko, W. Z. Zhang, K. Kimoto, Y. Matsui, Y. Taguchi, Y. Tokura, Nat. Commun. 2014, 5, 3198.

[24] X. Z. Yu, Y. Tokunaga, Y. Taguchi, Y. Tokura, Adv. Mater. 2017, 29, 1603958.

[25] W. H. Wang, Y. Zhang, G. Xu, L. Peng, B. Ding, Y. Wang, Z. Hou, X. Zhang, X. Li, E. Liu, S. Wang, J. Cai, F. Wang, J. Li, F. Hu, G. Wu, B. Shen, X. Zhang, Adv. Mater. 2016, 28, 6887.

[26] C. Phatak, O. Heinonen, M. D. Graef, A. Petford-Long, Nano Lett. 2016, 16, 4141.

[27] A. O. Leonov, M. Mostovoy, Nat. Commun. 2015, 6, 8275

[28] M. Pereiro, D. Yudin, H. Chico, C. Etz, O. Eriksson, A. Bergman, Nat. Commun. 2014, 5, 4815 .

[29] B. Malaman, D. Fruchart, G. L. Caër, J. Phys. F: Met. Phys. 1978, 8, 2389.

[30] G. L. Caër, B. Malaman, B. Roques, J. Phys. F: Met. Phys. 1978, 8, 323.

[31] G. L. Caër, B. Malaman, L. Häggström, T. Ericsson, J. Phys. F: Met. Phys. 1979, 9, 1905.

[32] L. A. Fenner, A. A. Dee, A. S. Wills, J. Phys.: Condens. Matter 2009, $21,452202$.

[33] T. Kida, L. A. Fenner, A. A. Dee, I. Terasaki, M. Hagiwara, A. S. Wills, J. Phys.: Condens. Matter 2011, 23, 112205

[34] Q. Wang, S. S. Sun, X. Zhang, F. Pang, H. C. Lei, Phys. Rev. B 2016, 94, 075135.

[35] H. F. Du, J. P. DeGrave, F. Xue, D. Liang, W. Ning, J. Y. Yang, M. L. Tian, Y. H. Zhang, S. Jin, Nano Lett. 2014, 14, 2026.

[36] T. Schulz, R. Ritz, A. Bauer, M. Halder, M. Waner, C. Frznz, C. Pfleiderer, K. Everschor, M. Garst, A. Rosch, Nat. Phys. 2012, 8, 301.

[37] W. J. Jiang, X. C. Zhang, G. Q. Yu, W. Zhang, X. Wang, M. B. Jungfleisch, J. E. Pearson, X. M. Cheng, O. Heinonen, K. L. Wang, Y. Zhou, A. Hoffmann, S. G. E. te Velthuis, Nat. Phys. 2017, 13, 162.

[38] K. Litzius, I. Lemesh, B. Krüger, P. Bassirian, L. Caretta, K. Richter, F. Büttner, K. Sato, O. A. Tretiakov, J. Förster, R. M. Reeve, M. Weigand, L. Bykova, H. Stoll, G. Schütz, G. S. D. Beach, M. Kläui, Nat. Phys. 2017, 13, 170

[39] S. Z. Lin, C. Reichhardt, C. D. Batista, A. Saxena, Phys. Rev. Lett. 2013, 110, 207202.

[40] C. Reichhardt, D. Ray, C. J. O. Reichhardt, Phys. Rev. B 2015, 91, 104426.

[41] M. J. Donahue, D. G. Porter, Object Oriented Micromagnetic Framework User's Guide Version 1.0 NISTIR 6376, National Institute of Standards and Technology, Gaithersburg, MD, USA 1999. 